\title{
Interaction of Silver Nanoparticles and Chitin Powder with Different Sizes and Surface Structures: The Correlation with Antimicrobial Activities
}

\author{
Vinh Quang Nguyen, ${ }^{1,2}$ Masayuki Ishihara, ${ }^{2}$ Shingo Nakamura, ${ }^{3}$ Hidemi Hattori, ${ }^{2}$ \\ Takeshi Ono, ${ }^{4}$ Yasushi Miyahira, ${ }^{4}$ and Takemi Matsui ${ }^{1}$ \\ ${ }^{1}$ Faculty of System Design, Tokyo Metropolitan University, 6-6 Asahigaoka, Hino, Tokyo 191-0065, Japan \\ ${ }^{2}$ Research Institute, National Defense Medical College, 3-2 Namiki, Tokorozawa, Saitama 359-1324, Japan \\ ${ }^{3}$ Department of Surgery, National Defense Medical College, 3-2 Namiki, Tokorozawa, Saitama 359-8513, Japan \\ ${ }^{4}$ Department of Global Infectious Diseases and Tropical Medicine, National Defense Medical College, 3-2 Namiki, \\ Tokorozawa, Saitama 359-8513, Japan \\ Correspondence should be addressed to Masayuki Ishihara; ishihara@ndmc.ac.jp
}

Received 10 July 2013; Revised 2 August 2013; Accepted 3 August 2013

Academic Editor: Jiamin Wu

Copyright ( 2013 Vinh Quang Nguyen et al. This is an open access article distributed under the Creative Commons Attribution License, which permits unrestricted use, distribution, and reproduction in any medium, provided the original work is properly cited.

\begin{abstract}
Silver nanoparticles (Ag NPs) were $5.17 \pm 1.9 \mathrm{~nm}$ in diameter, and four $<5 \%$ deacetylated chitins $(\mathrm{A}, \mathrm{B}, \mathrm{C}$, and $\mathrm{D})$ differing in size of powder and surface structure properties were used in the study. Chitin/Ag NP composites were synthesized by mixing Ag NP suspensions with each chitin powder at room temperature for $30 \mathrm{~min}$. The Ag NPs were homogenously dispersed and stably adsorbed onto the chitins A and B powders. The resulting chitin/Ag NP composites were brown; darker composites were obtained when larger amounts of Ag NPs were reacted with chitin. Approximately, 26 and $22 \mu \mathrm{g}$ of Ag NPs maximally adsorbed to $1 \mathrm{mg}$ of chitins A and B, respectively, whereas only 2.5 and $1.5 \mu \mathrm{g}$ of Ag NPs maximally adsorbed to chitins C and D, respectively. As the bactericidal and antifungal activities of the chitin/Ag NP composites increased with increasing amounts of Ag NPs adsorbed to the chitin, the antimicrobial activity of chitins $\mathrm{A}$ and $\mathrm{B} / \mathrm{Ag} \mathrm{NP}$ composites was much higher than that of chitins $\mathrm{C}$ and $\mathrm{D} / \mathrm{Ag}$ NP composites. These results suggest that the particle size and surface structure of the chitin powder critically influence both the adsorption and antimicrobial activity of Ag NPs.
\end{abstract}

\section{Introduction}

With the rise in microbial resistance to multiple antibiotics, considerable research has been carried out to develop effective antimicrobial agents free of resistance. This has led to resurgence in the use of silver- (Ag-) based antiseptics that have broad-spectrum antimicrobial activity and a much lower propensity to induce microbial resistance compared with antibiotics [1]. In fact, it is well known that Ag ions and Ag-based compounds are highly toxic to a broad range of microorganisms [2].

The chemical properties of Ag nanoparticles (Ag NPs) are significantly different from those of silver ingot or $\mathrm{Ag}$ ion, and thus Ag NPs have been studied by many researchers due to their wide variety of potential applications $[3,4]$. The special and unique properties of Ag NPs can be attributed to their smaller size and the larger specific surface area relative to bulk materials, and many preparation processes have been proposed for controlling the physical and/or chemical characteristics of Ag NPs [5-9].

Processes utilizing safe materials which do not require complicated purification steps can be employed to prepare $\mathrm{Ag}$ NPs for biomedical and environmental applications. It was reported that Ag NPs of less than $10 \mathrm{~nm}$ diameter can be produced through a process that employs D-glucose as the reducing agent and soluble starch as the stabilizing agent [10]. The size of Ag NPs can be controlled by modifying reaction system parameters such as $\mathrm{pH}$, temperature, and reactant concentrations. The choice of stabilizing agent is an important factor for controlling the size of Ag NPs because once 
they are generated, Ag NPs tend to fuse together and form aggregates that can grow to the size of microparticles [11].

The antimicrobial activity of zero-valent $\mathrm{Ag}$ is strictly depending on the surface development of the solid phase. When the solid phase is in a nanoparticle form, the antimicrobial activity of the resulting particles can be remarkably enhanced; as such, the antimicrobial activity of the smaller Ag NPs may be several orders of magnitude greater than that of the corresponding bulk solid. Therefore, adsorption of Ag NPs to the surface of various biomaterials could be a means of fabricating novel antimicrobial materials $[12,13]$.

Size is one of the most fundamental parameters affecting the optical [14], antimicrobial [15-17], and antiviral properties of Ag NPs [18, 19]. Sondi et al. reported that the antimicrobial activity of Ag NPs against Gram-negative bacteria such as Escherichia coli is dependent on particle concentration and that Ag NPs form "pits" in the bacterial cell wall, thus compromising its structural integrity [15]. Ag NPs also exhibit potent antifungal activity, probably through a similar mechanism involving compromise of membrane integrity [20].

On the other hand, there are some concerns about the biological and environmental risks associated with Ag NPs. It was reported that Ag NPs might adversely affect some aquatic organisms. For example, there are reports that $\mathrm{Ag}$ NPs may be cytotoxic and genotoxic to fish [21] and that Ag NPs may inhibit photosynthesis in algae [22]. Other evidence indicates that Ag NPs may also adversely affect mammals, as a significant decline in mouse spermatogonial stem cells was linked with exposure to Ag NPs [23]. Therefore, methods to prevent the diffusion of Ag NPs into the environment and inhibit their uptake by living organisms are needed before Ag NP-containing antimicrobial materials can be used [21-23].

In a previous study, we developed a safe and easy process for controlling the size distribution of Ag NPs [24]. The process uses three materials: $\mathrm{AgNO}_{3}$-containing glass powder, glucose, and water. Synthesis of Ag NPs using this process was performed by autoclaving the particles in aqueous medium. Particle size can be regulated by varying the glucose concentration; that is, $0.25,1.0$, and $4.0 \mathrm{wt} \%$ of glucose produce small $(3.48 \pm 1.83 \mathrm{~nm}$ in diameter $)$, medium $(6.63 \pm 1.78 \mathrm{~nm}$ in diameter $)$, and large $(12.9 \pm 2.5 \mathrm{~nm}$ in diameter $)$ particles, respectively [24]. Furthermore, chitin powder with low degrees of deacetylation (DDAc $<5 \%$ ) and with an average particle size of $250 \pm 70 \mu \mathrm{m}$ was added as a stabilizer to the Ag NP suspension to both remove the caramel generated during autoclaving and prevent aggregation and precipitation of the Ag NPs [25]. The much stronger bactericidal (against E. coli strain $\mathrm{DH} 5 \alpha$ ) and antifungal (against Aspergillus niger) activities of chitin/Ag NP composites have been demonstrated [25].

Chitin/chitosan is the collective name for a family of de- $N$-acetylated chitin with different degrees of deacetylation (DDAc) [26]. In general, when the number of $N$-acetylglucosamine units exceeds 50\% (DDAc > 50\%), the biopolymer is termed chitosan, whereas the term "chitin" is used to describe the polymer when the DDAc is less than $50 \%$. In this study, we observed that the antimicrobial activities and adsorption properties of Ag NPs are correlated with chitin particle size and surface structures of chitin powder.

\section{Materials and Methods}

2.1. Materials. Silver-containing glass powder (BSP21, Ag content: $1 \mathrm{wt} \%$; average grain size: $10 \mu \mathrm{m}$ ) was purchased from Kankyo Science (Kyoto, Japan). All chitins (A, B, C, and D) used in this study were $<5 \%$ DAc and were commercially available. D-Glucose was purchased from Wako Pure Chemical Industries, Ltd. (Osaka, Japan). All chemicals were used as received.

2.2. Preparation of Ag NPs. A suspension of size-controlled Ag NPs was prepared as previously described [24]. Briefly, $0.5 \mathrm{~g}$ of Ag-containing glass powder was dispersed in $50 \mathrm{~mL}$ of an aqueous solution of $0.8 \mathrm{wt} \%$ glucose in a $100 \mathrm{~mL}$ glass vial. The mixture was autoclaved at $121^{\circ} \mathrm{C}$ and $200 \mathrm{kPa}$ for $20 \mathrm{~min}$ and then gradually cooled to room temperature, after which it was centrifuged at $1,000 \mathrm{~g}$ for $10 \mathrm{~min}$. The resulting brown supernatant containing the Ag NP suspension was stored in the dark at $4^{\circ} \mathrm{C}$. The average diameter of Ag NPs prepared with $0.8 \mathrm{wt} \%$ glucose was $5.17 \pm 1.92 \mathrm{~nm}$, and the suspension contained about $60 \mu \mathrm{g} / \mathrm{mL}$ of Ag NPs.

2.3. Preparation of Chitin/Ag NP Composites. First, 20, 10, 5, $2.5,1.25$, or $0.6 \mathrm{mg}$ of chitin A (average particle size: $31 \pm$ $11 \mu \mathrm{m})$, chitin B $(104 \pm 42 \mu \mathrm{m})$, chitin $\mathrm{C}(894 \pm 121 \mu \mathrm{m})$, or chitin $\mathrm{D}(1,510 \pm 460 \mu \mathrm{m})$ (all chitins: $>5 \% \mathrm{DDAc}$ ) was added to $1 \mathrm{~mL}$ of Ag NP suspension (about $60 \mu \mathrm{g} / \mathrm{mL}$ ) and mixed well (at pH 7.2) for 30 min using a shaker (MildMixer PR-36; TAITEC, Tokyo, Japan). The chitin/Ag NP composites were washed twice with distilled water by centrifugation. The Ag NPs were homogenously dispersed and immobilized on the chitin powder. The composites were brown; darker composites were obtained when larger amounts of Ag NPs were reacted with each chitin. In addition, chitins $C$ and D were suspended in $0.2 \mathrm{M}$ acetate and mixed on the shaker for $18 \mathrm{~h}$. The resulting powders were washed twice with distilled water by centrifugation and allowed to air dry. The air-dried chitin $\mathrm{C}$ and $\mathrm{D}$ powders were ground thoroughly using a mortar and pestle to make the particles smaller, and then the ground chitin/Ag NP composites were produced as described above.

$\mathrm{UV}$-vis spectra were obtained at room temperature using a Jasco V-630 spectrophotometer (Jasco Corporation, Tokyo, Japan). Scanning electron microscopy (SEM) specimens of the chitins were coated with gold plasma to enhance conductivity using a plasma multicoater PMC-5000 (Meiwafosis Co., Ltd., Tokyo, Japan). SEM was performed using a JSM-6340F microscope (JEOL, Tokyo, Japan) operated at $5 \mathrm{kV}$.

Transmission electron microscopy (TEM) specimens were prepared by casting a small drop of a suspension of chitin/Ag NPs onto a carbon-coated copper grid; excess solution was then removed using filter paper, and the specimens were dried at room temperature. TEM images were obtained using a JEOL JEM-1010 microscope (Nihon Electronics Inc., Tokyo, Japan) operated at $80 \mathrm{kV}$.

2.4. Bactericidal Activity of Chitin/Ag NP Composites. A culture of E. coli strain DH5 $\alpha$ (Takara Co., Kyoto, Japan) was stored at $-80^{\circ} \mathrm{C}$ in Luria-Bertani (LB) broth containing $50 \%$ 

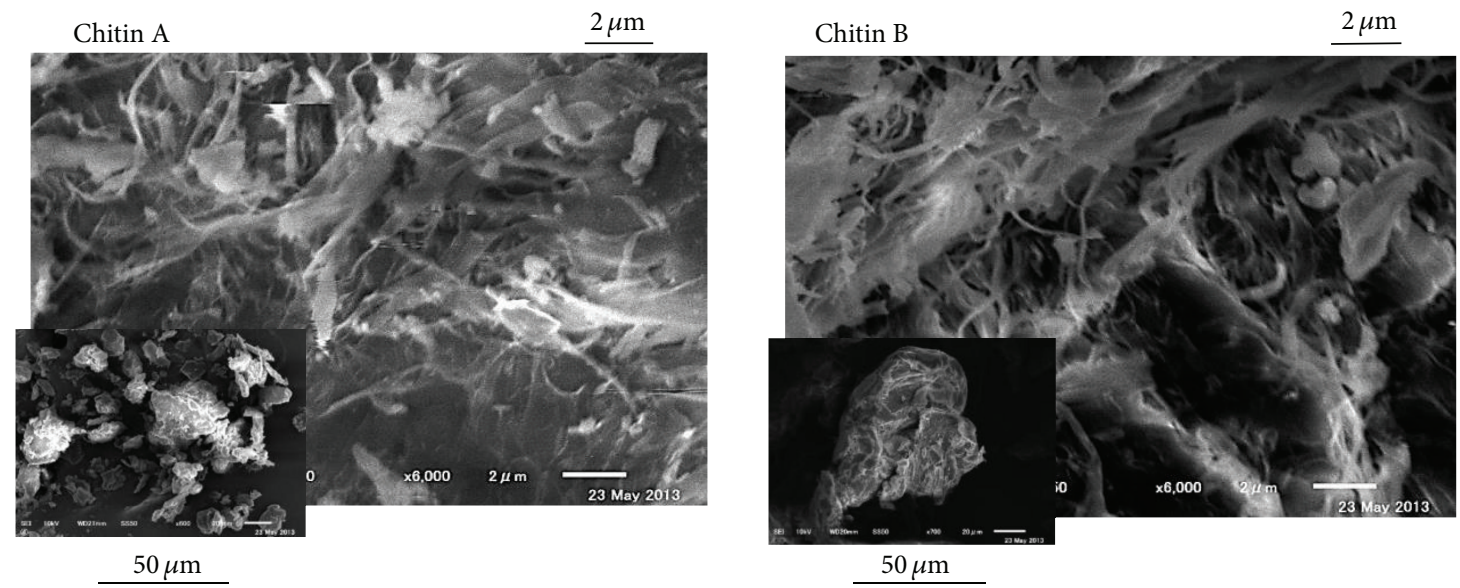

Chitin C

$2 \mu \mathrm{m}$

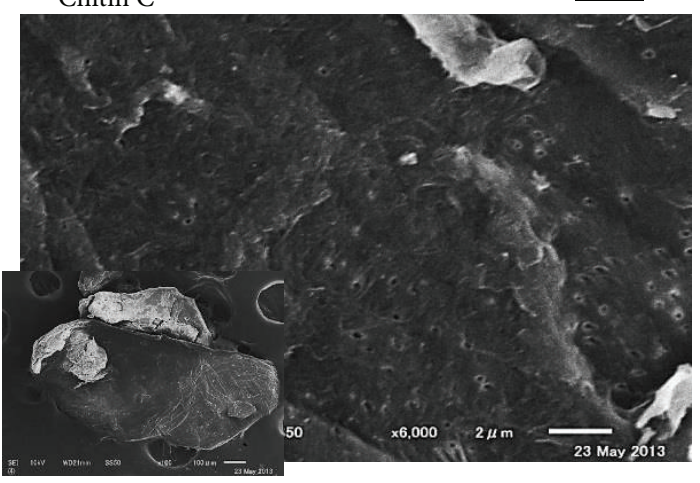

$200 \mu \mathrm{m}$

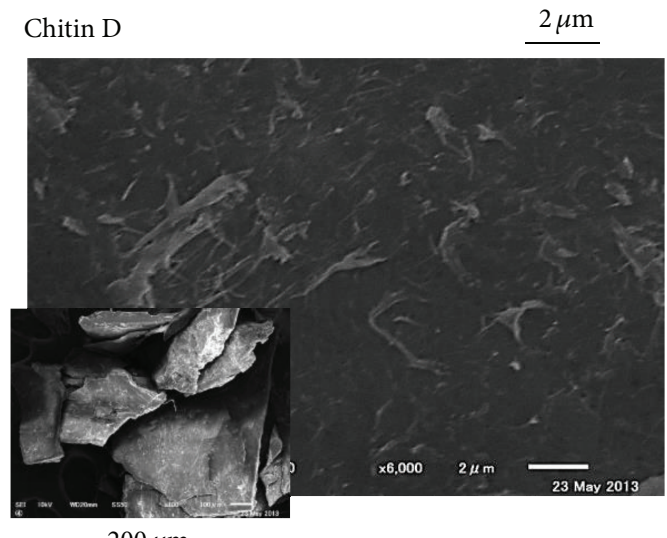

$200 \mu \mathrm{m}$

FIgURE 1: SEM micrographs of chitins A, B, C, and D.

sterile glycerol. Overnight cultures were prepared by growing a single E. coli colony overnight at $37^{\circ} \mathrm{C}$ in $5 \mathrm{~mL}$ of $\mathrm{LB}$ medium. On the next day, $200 \mu \mathrm{L}$ of the overnight culture was inoculated into $2 \mathrm{~mL}$ of $\mathrm{LB}$ medium and incubated at $37^{\circ} \mathrm{C}$ for $6 \mathrm{~h}$ or until the optical density at $600 \mathrm{~nm}\left(\mathrm{OD}_{600}\right)$ reached 0.260 . The $E$. coli culture was then diluted 4 -fold with $\mathrm{LB}$ broth, after which $50 \mu \mathrm{L}$ of the diluted suspension was added to separate sterile 1.5-mL ClickFit polypropylene microcentrifuge tubes (TreffLab AG, Degersheim, Switzerland) containing dried chitin/Ag NP composites with 2, 1, 0.5, or $0 \mu \mathrm{g}$ of Ag NPs adsorbed to $10 \mathrm{mg}$ of each chitin type. The samples were then incubated at $37^{\circ} \mathrm{C}$ for $18 \mathrm{~h}$, after which $1 \mathrm{~mL}$ of LB medium was added to each suspension and mixed well. The suspensions were allowed to stand for $3 \mathrm{~min}$ to precipitate the chitin/Ag NP composites. Viable cells were enumerated by plating $50 \mu \mathrm{L}$ of 10 -fold serial dilutions of the suspensions onto LB agar (ForMedium Ltd., Hunstanton, UK) in a $90 \times$ $15 \mathrm{~mm}$ Petri dish, followed by incubation at $37^{\circ} \mathrm{C}$ for $24 \mathrm{~h}$.

2.5. Antifungal Activity of the Ag NP/Chitin Composites. Aspergillus niger NBRC105649 (Japan Collection of Microorganisms; Wako, Saitama, Japan) was maintained in molten potato dextrose agar (PDA) medium (Difco, Becton Dickinson \& Co., Sparks, MD, USA). Aliquots $(20 \mu \mathrm{L})$ of a suspension of $A$. niger spores $\left(6.35 \times 10^{4}\right.$ spores $\left./ \mathrm{mL}\right)$ were inoculated into each well of a 24 -well plate (well diameter:
17 mm; Sumitomo Bakelite Co., Ltd., Tokyo, Japan) containing $1 \mathrm{~mL}$ of PDA prepared with $60,30,15$, or $7.5 \mu \mathrm{g} / \mathrm{mL}$ of $\mathrm{Ag}$ NPs adsorbed onto $5 \mathrm{mg}$ of each chitin. The plates were incubated in the dark at $25^{\circ} \mathrm{C}$ for 3 days, after which the $A$. niger spores were recovered into $500 \mu \mathrm{L}$ of $0.3 \%$ sterile Tween 80 solution using a platinum loop. After vortexing, the absorbance of each spore suspension was measured at $550 \mathrm{~nm}$ using a Jasco V-630 spectrophotometer [25].

\section{Results and Discussion}

3.1. Characterization of the Chitin/Ag NP Composites. In this work, $<5 \%$ DAc chitins A, B, C, ground C (G-C), D and ground $D(G-D)$ with various particle sizes and with various surface structures were added to the Ag NP suspensions to remove the caramel produced during autoclaving and to prevent aggregation and precipitation of the Ag NPs. The Ag NPs were adsorbed tightly to chitins A and B by just mixing at $\mathrm{pH} 7.0$ for $30 \mathrm{~min}$. The chitins $\mathrm{A}$ and B/Ag NP composites were substantially more stable than Ag NPs alone. Caramel was removed from the chitin/Ag NP composites by washing them twice with distilled water. The caramel had to be removed because of its inadequate growth effect of microbial growth.

Typical SEM micrographs of chitins A, B, C, and D are shown in Figure 1. Chitins A and B exhibited smaller powder 

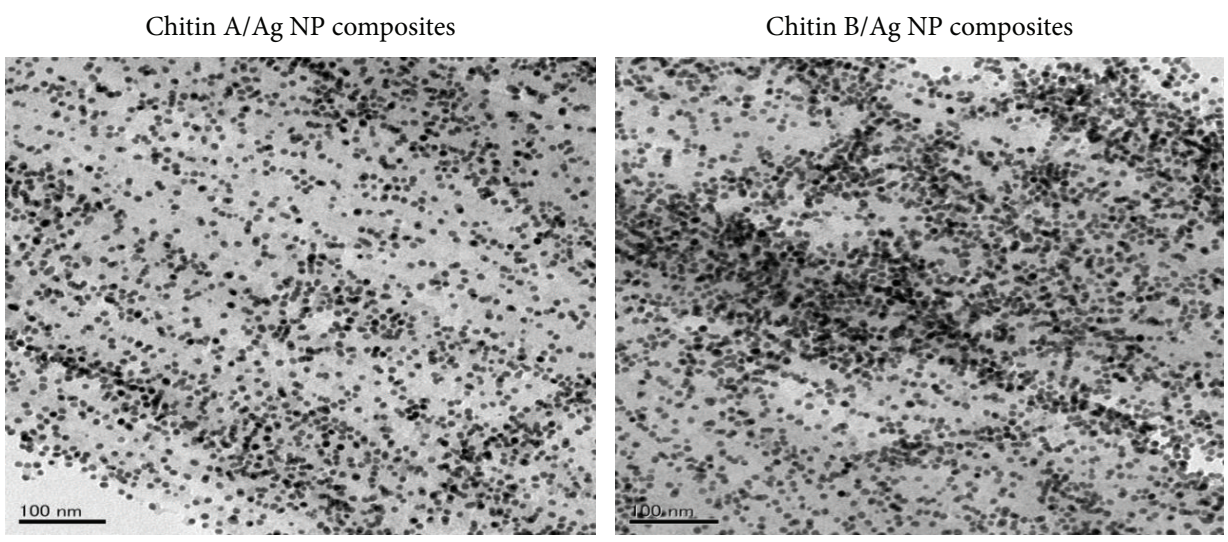

Chitin C/Ag NP composites
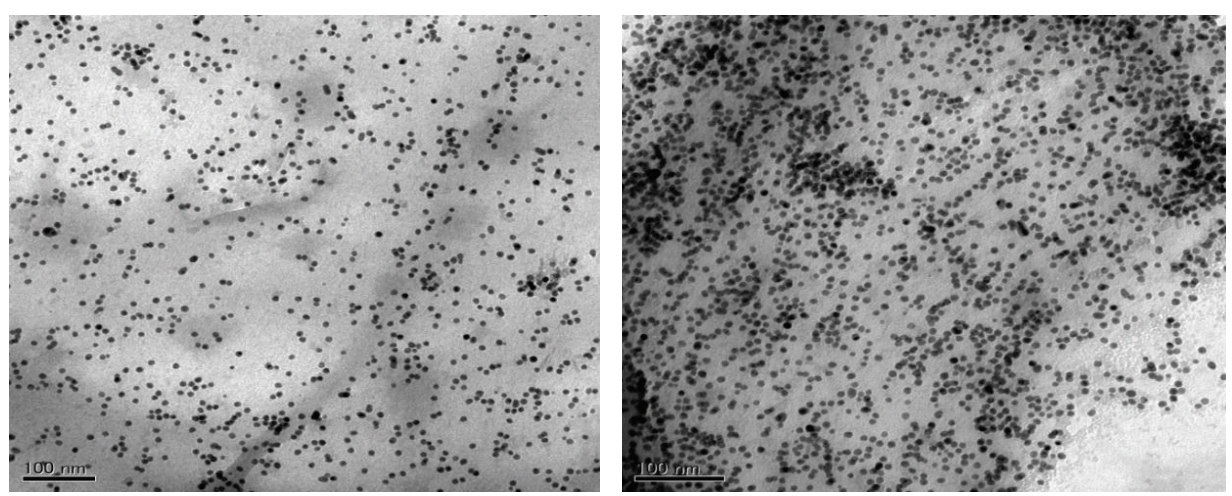

Chitin D/Ag NP composites

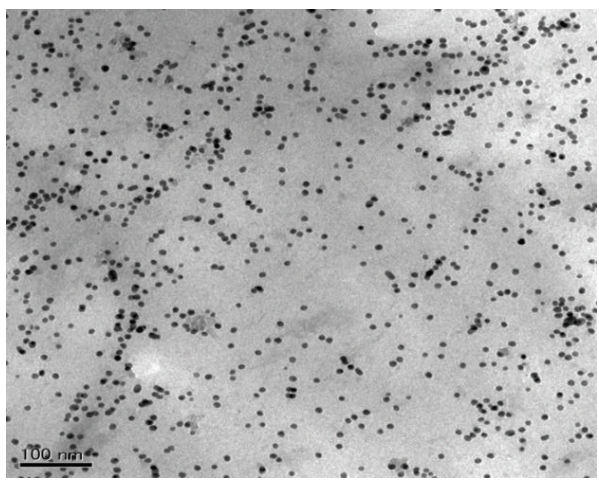

Chitin G-D/Ag NP composites

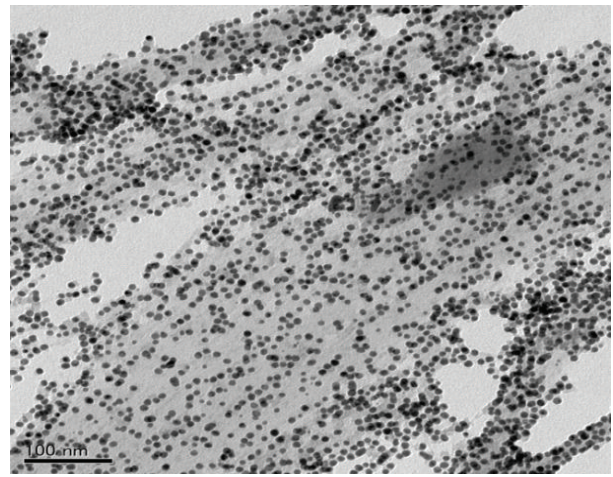

$100 \mathrm{~nm}$

Figure 2: TEM micrographs of chitins A, B, C, G-C, D, and G-D/Ag NP composites $(\times 250,000)$. A total of 1 mg of each chitin were added to a $10 \mu \mathrm{g} / \mathrm{mL}$ suspension of Ag NPs and mixed for $30 \mathrm{~min}$ prior to analysis.

particle size and a nanoscale fiber-like surface structure, whereas chitins $C$ and $D$ showed a larger powder particle size and a flat/smooth film-like surface structure. Chitins G-C and G-D exhibited a smaller powder particle size and a nanoscale fiber-like surface structure similar to that of chitins A and B (data not shown). The powder sizes and shapes of the Ag NPs adsorbed to all of the chitins examined were identical to those of the original Ag NPs used for synthesizing the composites (Figure 2). The average particle size of chitin G-C and chitin G-D was $146 \pm 62$ and $181 \pm 52 \mu \mathrm{m}$, respectively
(Table 1), indicating that larger chitin powder particles with a flat/smooth film-like surface structure can be changed into smaller particles with a nanoscale fiber-like surface structure by grinding. The color of the composites was brown; a darker composite was obtained when larger amounts of Ag NPs were adsorbed to the chitin.

As shown in Table 1, approximately 26, 22, 2.5, 20, 1.5 and $18 \mu \mathrm{g}$ of Ag NPs maximally adsorbed to $1 \mathrm{mg}$ of chitins A, B, C, G-C, D, and G-D, respectively. Figures 3 and 4 show UV-vis spectra of Ag NPs in suspension and spectra of the 

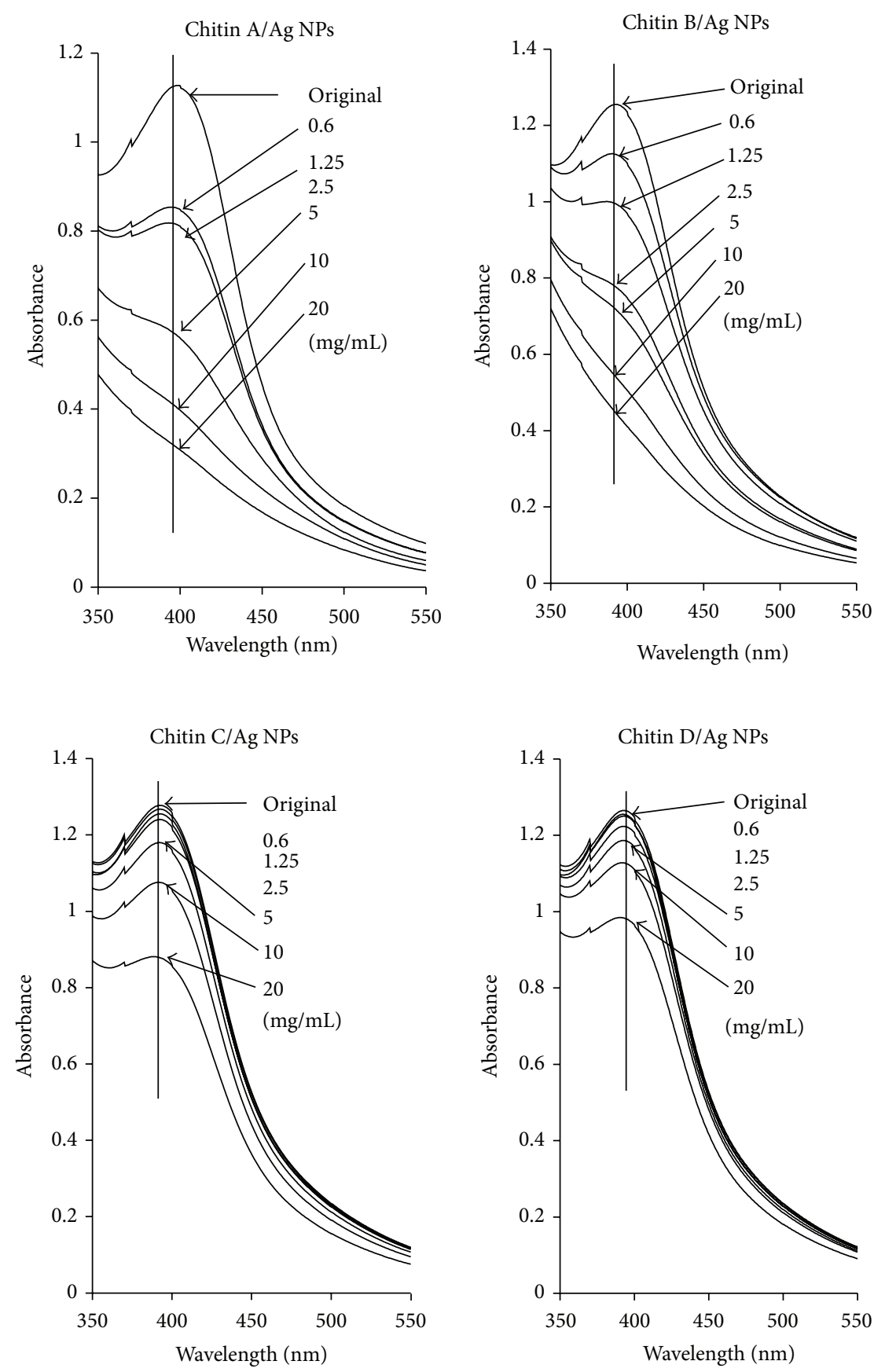

FIGURE 3: UV-vis spectra of original Ag NPs in suspension (original) and supernatants from the postreaction mixtures in which various amounts of chitins A, B, C, and D reacted with Ag NPs. Excess Ag NPs in the supernatants of the postreaction mixtures decreased as the amount of chitin added increased.

supernatants of the postreaction mixtures in which various amounts of chitin reacted with the Ag NPs. The peak at $390.5 \mathrm{~nm}$ is representative of the spherical Ag NPs used in this work $[12,24]$. There was a proportional relationship between the absorbance at $390.5 \mathrm{~nm}$ and the concentration of Ag NPs in the suspension [25]. The amount of Ag NPs remaining in the supernatants of the postreaction mixtures decreased as the concentration of chitin in the reaction mixture increased (Figures 3 and 4). Thus, Ag NPs selectively reacted with chitins G-C and G-D in addition to chitins A and B.
3.2. Antimicrobial Activity of the Chitin/Ag NP Composites. The antimicrobial activity of Ag NPs has been demonstrated in a number of studies [13-15, 18, 20]. Investigation of this phenomenon has gained importance due to the rise in resistance to antibiotics caused by their overuse. The bactericidal activity of each chitin type alone and each chitin/Ag NP composite was evaluated against $E$. coli. The individual chitins alone exhibited only weak bactericidal activity. Composites with various amounts of $\mathrm{Ag}$ NPs in $10 \mathrm{mg} / \mathrm{mL}$ of chitins $\mathrm{A}$, B, G-C, or G-D showed strong, concentration-dependent 

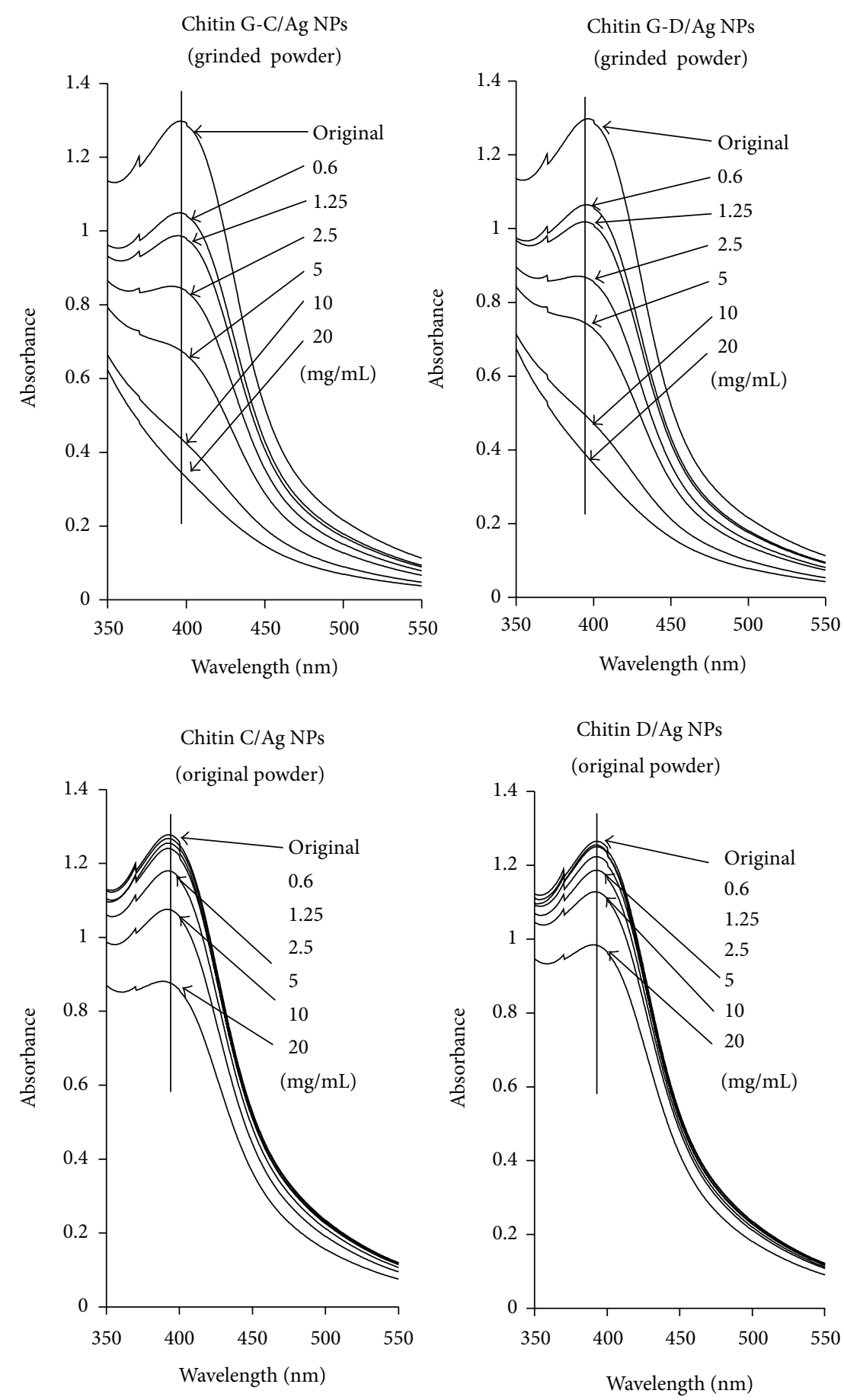

FIGURE 4: UV-vis spectra of original Ag NPs in suspension (original) and supernatants from the postreaction mixtures in which various amounts of chitins G-C, G-D, C, and D reacted with Ag NPs. Excess Ag NPs in the supernatants of the postreaction mixtures decreased as the amount of chitin added increased.

bactericidal activity, whereas the chitins $\mathrm{C}$ and $\mathrm{D} / \mathrm{Ag} \mathrm{NP}$ composites demonstrated only weak bactericidal activity because of lower interactions between the chitin and Ag NPs (Table 1 and Figure 5).

The antifungal activity of each chitin type alone and each chitin/Ag NP composite was evaluated against $A$. niger. Fungi were incubated in molten PDA containing the test materials. Chitin alone ( $5 \mathrm{mg} / \mathrm{mL}$ ) exhibited weak antifungal activity (Figure 6). When composites with various amounts of $\mathrm{Ag}$ NPs in $5 \mathrm{mg} / \mathrm{mL}$ of chitin were added to the fungal cultures in PDA, composites containing chitins A, B, GC, and G-D showed strong, concentration-dependent antifungal activity, with half-growth inhibition occurring at $\mathrm{Ag}$ NP concentrations of $10,12,14$, and $15 \mu \mathrm{g} / \mathrm{mL}$, respectively 


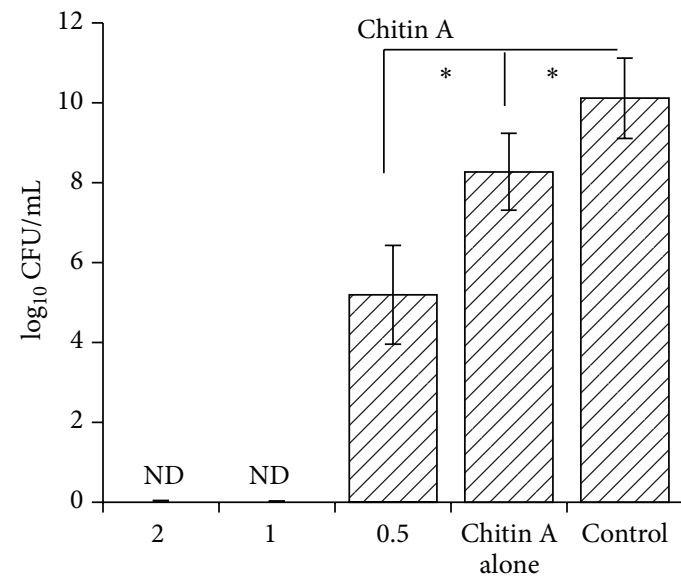

Concentration of Ag NPs $(\mu \mathrm{g} / \mathrm{mL})$ in $10 \mathrm{mg}$ chitin

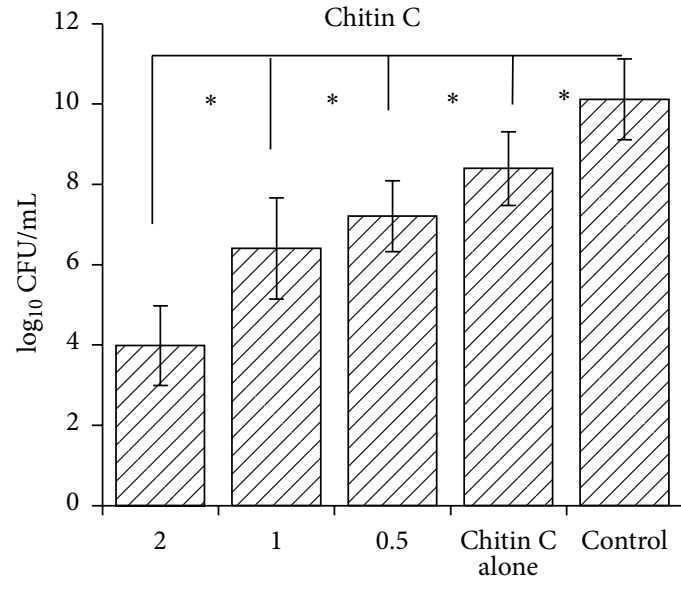

Concentration of Ag NPs $(\mu \mathrm{g} / \mathrm{mL})$ in $10 \mathrm{mg}$ chitin

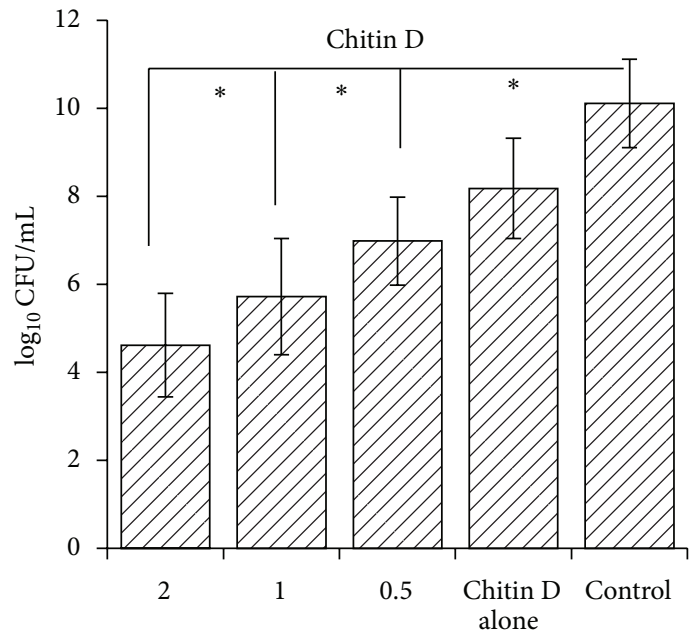

Concentration of Ag NPs $(\mu \mathrm{g} / \mathrm{mL})$ in $10 \mathrm{mg}$ chitin

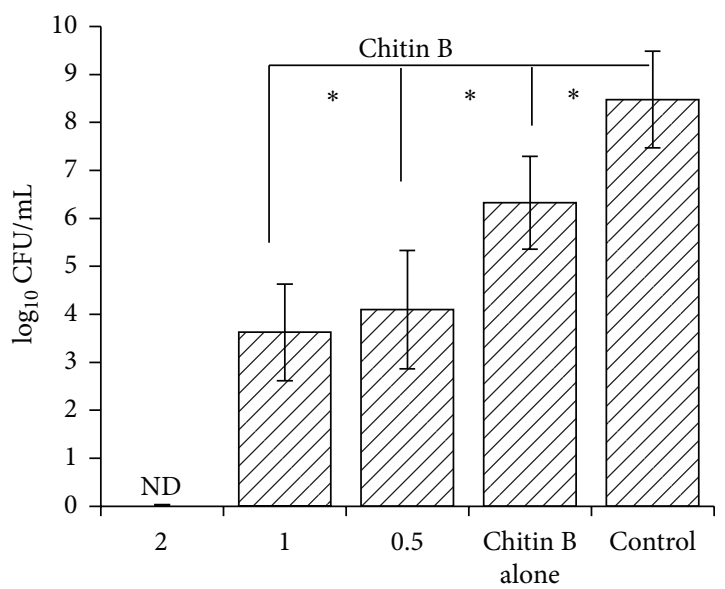

Concentration of Ag NPs $(\mu \mathrm{g} / \mathrm{mL})$ in $10 \mathrm{mg}$ chitin

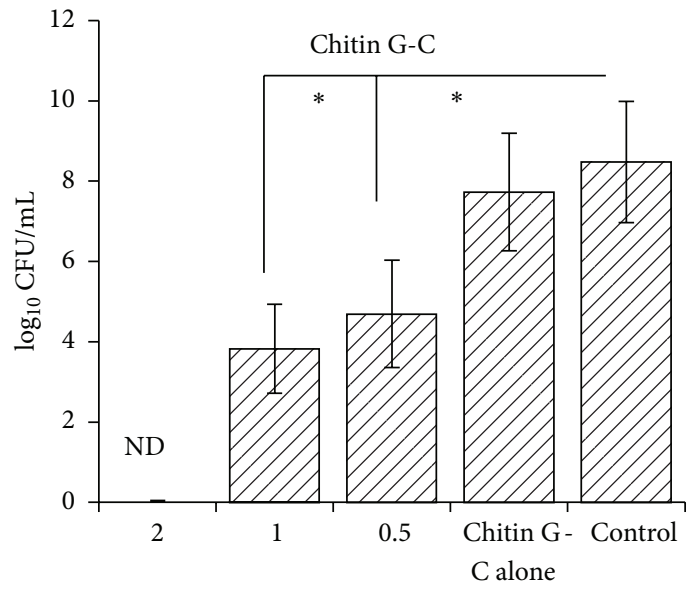

Concentration of Ag NPs $(\mu \mathrm{g} / \mathrm{mL})$ in $10 \mathrm{mg}$ chitin

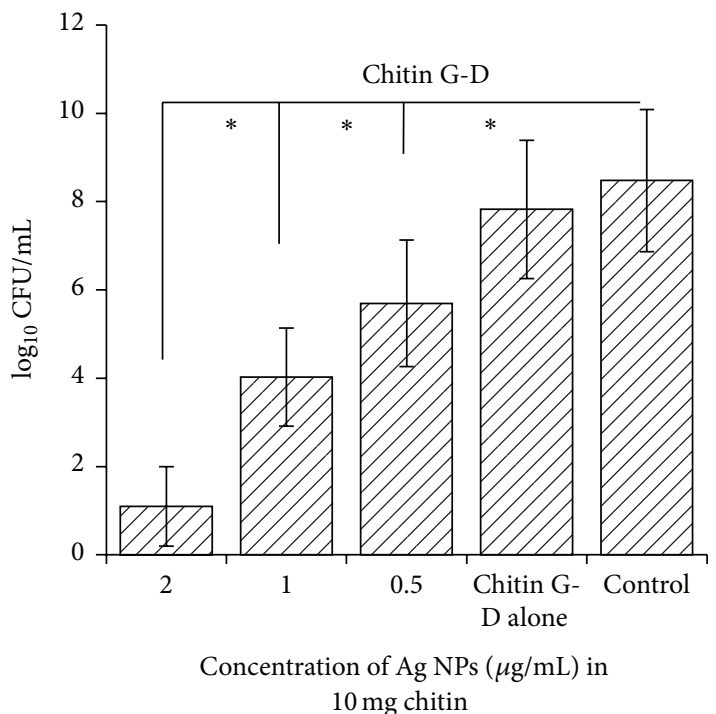

Figure 5: Chitins A, B, C, G-C, D, and G-D/Ag NP composites were evaluated for their bactericidal (against E. coli) activity in LB medium. The composites contained various amounts of Ag NPs in $10 \mathrm{mg}$ of chitin and exhibited a strong, concentration-dependent bactericidal activity. Data are the mean \pm standard deviation; $n=6$. ND $=$ not detected. Asterisks $(*)$ denote statistically significant differences $(P<0.01)$ as determined using a two-sample $t$-test. 
TABLE 1: Evaluation of each chitin/Ag NP composites.

\begin{tabular}{lccc}
\hline & $\begin{array}{c}\text { Maximal amount of Ag NPs }(\mu \mathrm{g}) \\
\text { absorbed in 1 mg chitin }(\mu \mathrm{g} / \mathrm{mg})\end{array}$ & $\begin{array}{c}\text { Half-bactericidal activity with } \\
\log _{10} \text { CFU/mL for Ag NPs }(\mu \mathrm{g}) \\
10 \mathrm{mg} \text { chitin }(\mu \mathrm{g} / 10 \mathrm{~mL})\end{array}$ & $\begin{array}{c}\text { Half-antifungal activity for Ag NPs } \\
(\mu \mathrm{g}) \text { in } 5 \mathrm{mg} \text { chitin }(\mu \mathrm{g} / 5 \mathrm{~mL})\end{array}$ \\
\hline Chitin A & 26 & 0.5 & 10 \\
Chitin B & 22 & 0.5 & 12 \\
Chitin C & 2.5 & 2.0 & $>60$ \\
Chitin G-C & 20 & 0.5 & 14 \\
Chitin D & 1.5 & $>2.0$ & $>60$ \\
Chitin G-D & 18 & 0.5 & 15 \\
\hline
\end{tabular}

Chitin A, powder size: average $31 \mu \mathrm{m}$, nanofiber-like structures.

Chitin B, powder size: average $104 \mu \mathrm{m}$, nanofiber-like structures.

Chitin C, powder size: average $894 \mu \mathrm{m}$, smooth film-like structures.

Chitin G-C, powder size: average $146 \mu \mathrm{m}$, nanofiber-like structures.

Chitin D, powder size: average $>1500 \mu \mathrm{m}$, smooth film-like structures.

Chitin G-D powder size: average $181 \mu \mathrm{m}$, nanofiber-like structures.

Ag NPs: average diameter $5.17 \pm 1.9 \mathrm{~nm}, \mathrm{G}$-: ground.

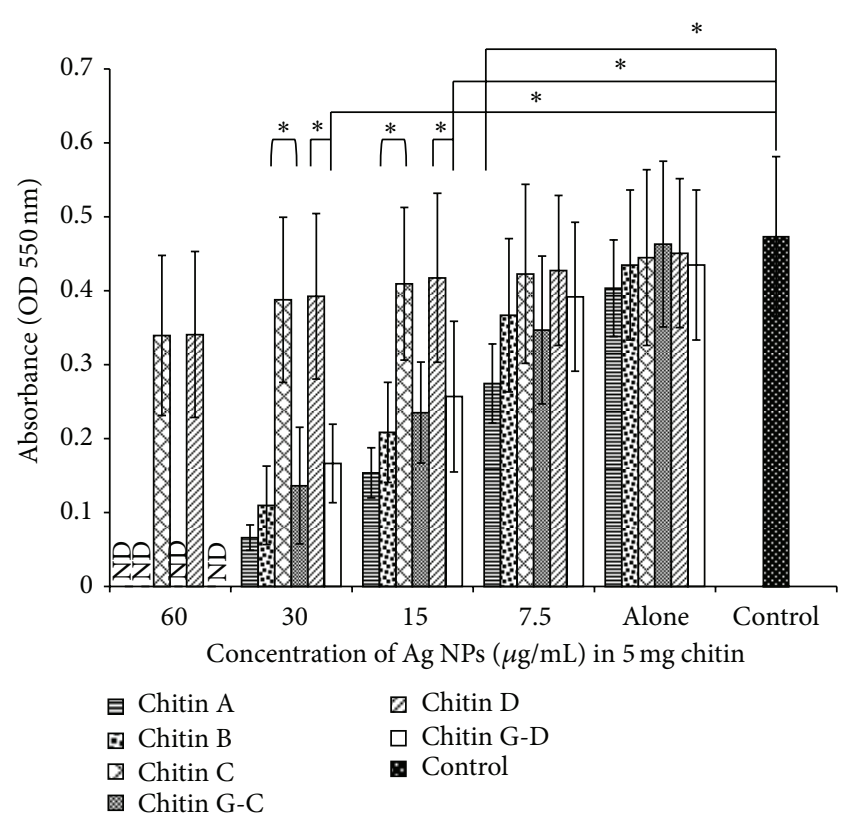

Figure 6: Chitins A, B, C, G-C, D, and G-D/Ag NP composites were evaluated for their antifungal activity against $A$. niger by incubating fungal spores on PDA with composites consisting of various amounts of Ag NPs in $5 \mathrm{mg} / \mathrm{mL}$ suspensions of each chitin. The composites exhibited a strong Ag NP concentration-dependent antifungal activity. Data are the mean \pm standard deviation; $n=6$. Asterisks $(*)$ denote statistically significant differences $(P<0.01)$ as determined using a two-sample $t$-test.

(Figure 6). Composites containing chitins $\mathrm{C}$ and D exhibited only weak antifungal activity. These results demonstrated that the antifungal activity of chitin/Ag NP composites increases as the amount of Ag NPs adsorbed to the chitin particles increases. The antimicrobial activity of Ag NPs adsorbed onto the smaller chitin powder particles with a nanoscale fiberlike surface structure was enhanced for two main reasons: (i) the available binding area for Ag NPs increases with decreasing chitin powder particle size together with the presence of the nanoscale fiber-like surface structure; (ii) chitin/Ag NP composites with higher amounts of Ag NPs are able to interact with microorganisms more efficiently. The sizes and morphologies of bound Ag NPs onto each chitin were maintained for at least 3 months.

The mechanism of the bactericidal action of $\mathrm{Ag}$ ions is closely related to their interaction with proteins, particularly with thiol groups (sulfhydryl, - SH), which is believed to be bridged by Ag ions, thus binding protein molecules together and disrupting their function. Because many proteins function as enzymes, disruption of function leads to a breakdown in cellular metabolism, followed by death $[13,14]$. However, the bactericidal and antifungal mechanisms of Ag NPs have not been investigated extensively. It has been reported that the activity of Ag NPs against gram-negative bacteria such as $E$. coli is dependent on the Ag NP concentration and involves the formation of "pits" in the bacterial cell wall [13]. The bactericidal activity of Ag NPs (against E. coli) is likely due to direct binding of the microbial envelope glycoproteins by the Ag NPs adsorbed to the nanoscale fiber-like surface structures of the small chitin powder particles, facilitating an interaction with the membrane that compromises its integrity. The potent antifungal activity (against $A$. niger) of the chitin/Ag NP composites is also probably related to compromise of membrane integrity [20]. In large chitin/Ag NP composites containing lower amounts of Ag NPs, spatial restriction due to the larger size of the chitin powder particles may prevent or weaken the interaction between microorganisms and the Ag NPs. In contrast, composites prepared with small chitin powder particles and with higher amounts of Ag NPs appear to interact more efficiently with the microbial cell surface [25].

\section{Conclusion}

In this work, various chitins with small powder particle sizes and nanoscale fiber-like surface structures were used to stabilize Ag NPs in suspensions and remove the caramel generated during autoclaving, thus preventing aggregation and precipitation of the Ag NPs. The Ag NPs were homogenously 
dispersed and stably adsorbed onto nanoscale fiber-like surface of the smaller chitin powder particles. The bactericidal and antifungal activities of the chitin/Ag NP composites we examined increased as the amount of Ag NPs adsorbed to the chitin particles increased. These results demonstrate the potential applications of small chitin powders with nanoscale fiber-like surface structures as a novel stabilizer and carrier for Ag NPs. Furthermore, chitin/Ag NP composites could be used directly as antimicrobial materials.

\section{Conflict of Interests}

The authors state that they have no conflict of interests.

\section{Acknowledgment}

This study was carried out using commercially available materials and equipment. The authors would like thank Ms. Y. Ichiki from the Laboratory Center of the National Defense Medical College (Tokorozawa, Japan) for help with electron microscopy experiments.

\section{References}

[1] D. J. Anderson and M. Moskovits, "A SERS-active system based on silver nanoparticles tethered to a deposited silver film," Journal of Physical Chemistry B, vol. 110, no. 28, pp. 13722-13727, 2006.

[2] A. Kumar, P. K. Vemula, P. M. Ajayan, and G. John, "Silvernanoparticle-embedded antimicrobial paints based on vegetable oil," Nature Materials, vol. 7, no. 3, pp. 236-241, 2008.

[3] S. Sharma, N. Ahmad, A. Prakash, V. N. Singh, A. K. Ghoash, and B. R. Mehta, "Synthesisof crystalline AG nanoparticles, (Ag NPs) from microorganisms," Materials Sciences and Applications, vol. 1, pp. 1-7, 2010.

[4] W. Ngeontae, W. Janrungroatsakul, P. Maneewattanapinyo, S. Ekgasit, W. Aeungmaitrepirom, and T. Tuntulani, "Novel potentiometric approach in glucose biosensor using silver nanoparticles as redox marker," Sensors and Actuators B, vol. 137, no. 1, pp. 320-326, 2009.

[5] S. Komarneni, D. Li, B. Newalkar, H. Katsuki, and A. S. Bhalla, "Microwave-polyol process for Pt and Ag nanoparticles," Langmuir, vol. 18, no. 15, pp. 5959-5962, 2002.

[6] S. Navaladian, B. Viswanathan, R. P. Viswanath, and T. K. Varadarajan, "Thermal decomposition as route for silver nanoparticles," Nanoscale Research Letters, vol. 2, no. 1, pp. 44-48, 2007.

[7] N. Vigneshwaran, N. M. Ashtaputre, P. V. Varadarajan, R. P. Nachane, K. M. Paralikar, and R. H. Balasubramanya, "Biological synthesis of silver nanoparticles using the fungus Aspergillus flavus," Materials Letters, vol. 61, no. 6, pp. 1413-1418, 2007.

[8] M. Z. Kassaee, A. Akhavan, N. Sheikh, and R. Beteshobabrud, " $\gamma$-Ray synthesis of starch-stabilized silver nanoparticles with antibacterial activities," Radiation Physics and Chemistry, vol. 77, no. 9, pp. 1074-1078, 2008.

[9] G. N. Xu, X. L. Qiao, X. L. Qiu, and J. G. Chen, "Preparation and characterization of stable monodisperse silver nanoparticles via photoreduction," Colloids and Surfaces A, vol. 320, no. 1-3, pp. 222-226, 2008.

[10] P. Raveendran, J. Fu, and S. L. Wallen, "Completely "Green" Synthesis and Stabilization of Metal Nanoparticles," Journal of the American Chemical Society, vol. 125, no. 46, pp. 13940-13941, 2003.

[11] N. Vigneshwaran, R. P. Nachane, R. H. Balasubramanya, and P. V. Varadarajan, "A novel one-pot "Green" synthesis of stable silver nanoparticles using soluble starch," Carbohydrate Research, vol. 341, no. 12, pp. 2012-2018, 2006.

[12] I. O. Sosa, C. Noguez, and R. G. Barrera, "Optical properties of metal nanoparticles with arbitrary shapes," Journal of Physical Chemistry B, vol. 107, no. 26, pp. 6269-6275, 2003.

[13] I. Sondi and B. Salopek-Sondi, "Silver nanoparticles as antimicrobial agent: a case study on E. coli as a model for Gramnegative bacteria," Journal of Colloid and Interface Science, vol. 275, no. 1, pp. 177-182, 2004.

[14] J. R. Morones, J. L. Elechiguerra, A. Camacho et al., "The bactericidal effect of silver nanoparticles," Nanotechnology, vol. 16, no. 10, pp. 2346-2353, 2005.

[15] X. L. Cao, C. Cheng, Y. L. Ma, and C. S. Zhao, "Preparation of silver nanoparticles with antimicrobial activities and the researches of their biocompatibilities," Journal of Materials Science, vol. 21, no. 10, pp. 2861-2868, 2010.

[16] P. Pallavicini, A. Taglietti, G. Dacarro et al., "Self-assembled monolayers of silver nanoparticles firmly grafted on glass surfaces: low $\mathrm{Ag}+$ release for an efficient antibacterial activity," Journal of Colloid and Interface Science, vol. 350, no. 1, pp. 110116, 2010.

[17] P. Dallas, V. K. Sharma, and R. Zboril, "Silver polymeric nanocomposites as advanced antimicrobial agents: classification, synthetic paths, applications, and perspectives," Advances in Colloid and Interface Science, vol. 166, no. 1-2, pp. 119-135, 2011.

[18] J. L. Elechiguerra, J. L. Burt, J. R. Morones et al., "Interaction of silver nanoparticles with HIV-1," Journal of Nanobiotechnology, vol. 3, p. 6, 2005.

[19] H. H. Lara, N. V. Ayala-Nuñez, L. Ixtepan-Turrent, and C. Rodriguez-Padilla, "Mode of antiviral action of silver nanoparticles against HIV-1," Journal of Nanobiotechnology, vol. 8, p. 1, 2010.

[20] K. J. Kim, W. S. Sung, B. K. Suh et al., "Antifungal activity and mode of action of silver nano-particles on Candida albicans," BioMetals, vol. 22, no. 2, pp. 235-242, 2009.

[21] J. P. Wise, B. C. Goodale, S. S. Wise et al., "Silver nanospheres are cytotoxic and genotoxic to fish cells," Aquatic Toxicology, vol. 97, no. 1, pp. 34-41, 2010.

[22] E. Navarro, F. Piccapietra, B. Wagner et al., "Toxicity of silver nanoparticles to Chlamydomonas reinhardtii," Environmental Science and Technology, vol. 42, no. 23, pp. 8959-8964, 2008.

[23] L. K. Braydich-Stolle, B. Lucas, A. Schrand et al., "Silver nanoparticles disrupt GDNF/Fyn kinase signaling in spermatogonial stem cells," Toxicological Sciences, vol. 116, no. 2, pp. 577-589, 2010.

[24] Y. Mori, T. Tagawa, M. Fujita et al., "Simple and environmentally friendly preparation and size control of silver nanoparticles using an inhomogeneous system with silver-containing glass powder," Journal of Nanoparticle Research, vol. 13, no. 7, pp. 2799-2806, 2011.

[25] V. Q. Nguyen, M. Ishihara, Y. Mori et al., "Preparation of sizecontrolled silver nanoparticles and Chitin-based composites and their antimicrobial activities," Journal of Nanomaterials, vol. 2013, Article ID 693486, 7 pages, 2013.

[26] C. Shi, Y. Zhu, X. Ran, M. Wang, Y. Su, and T. Cheng, "Therapeutic potential of chitosan and its derivatives in regenerative medicine," Journal of Surgical Research, vol. 133, no. 2, pp. 185192, 2006. 

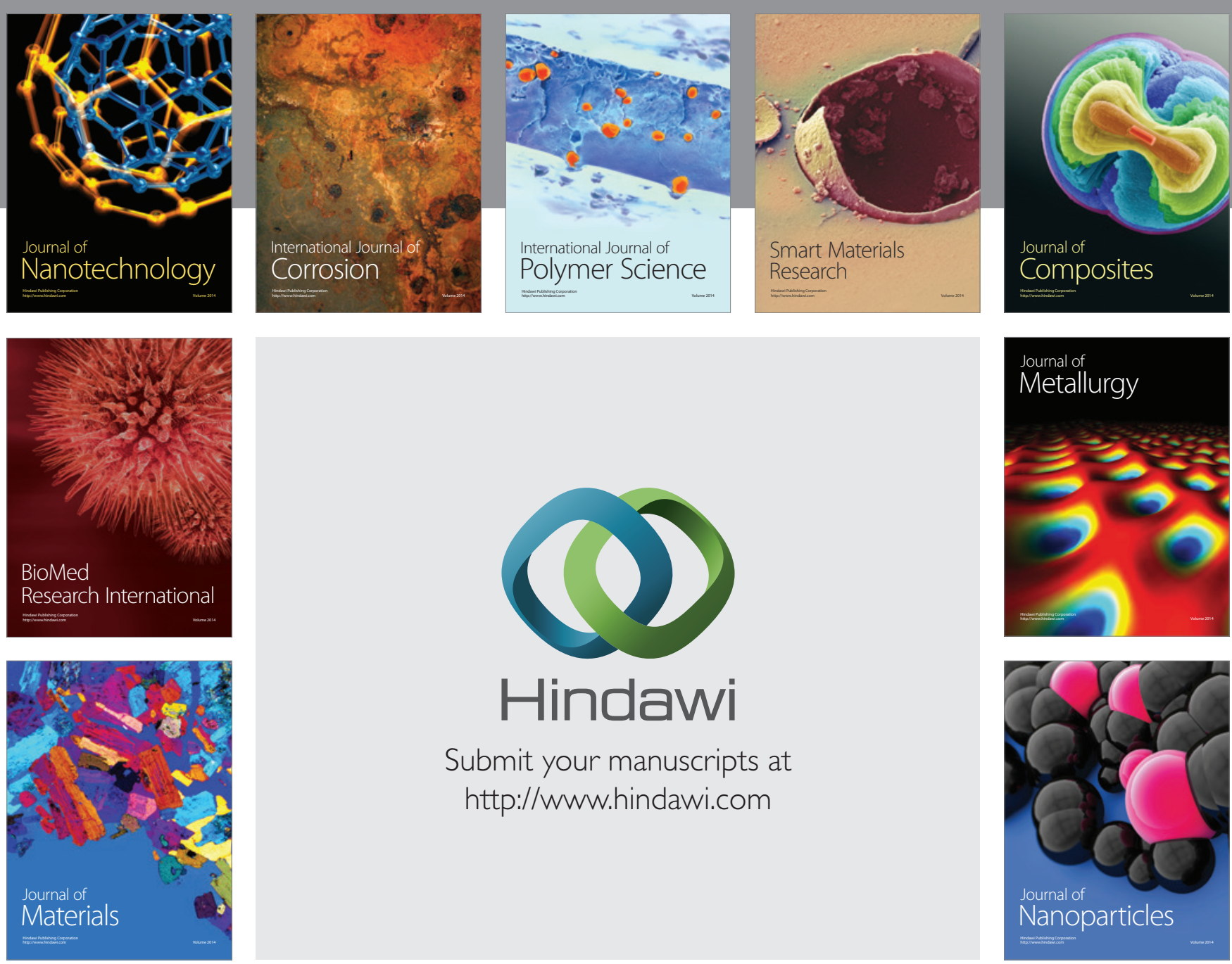

Submit your manuscripts at http://www.hindawi.com
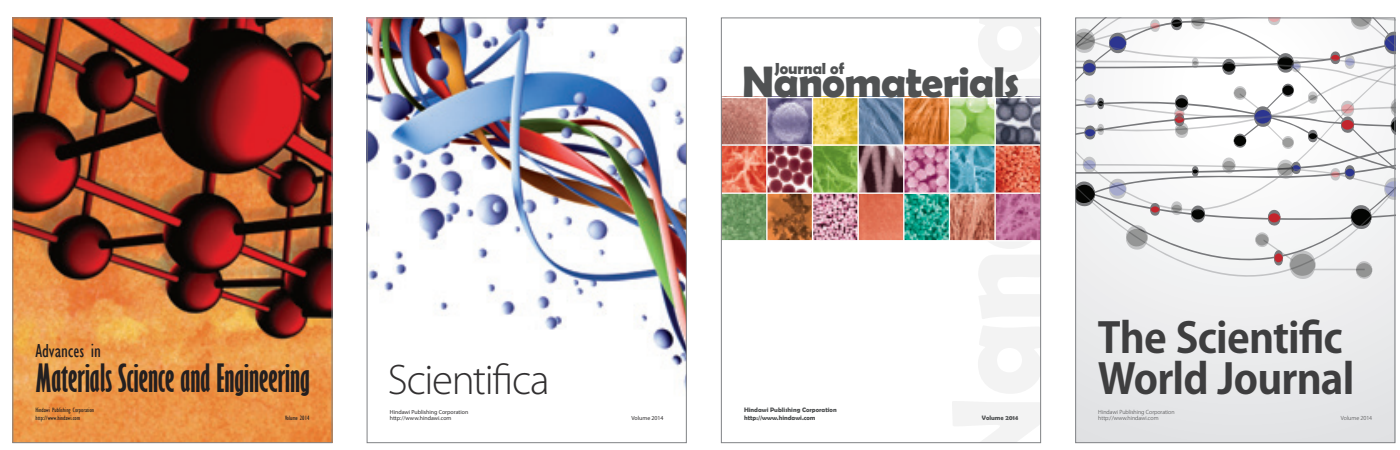

\section{The Scientific World Journal}
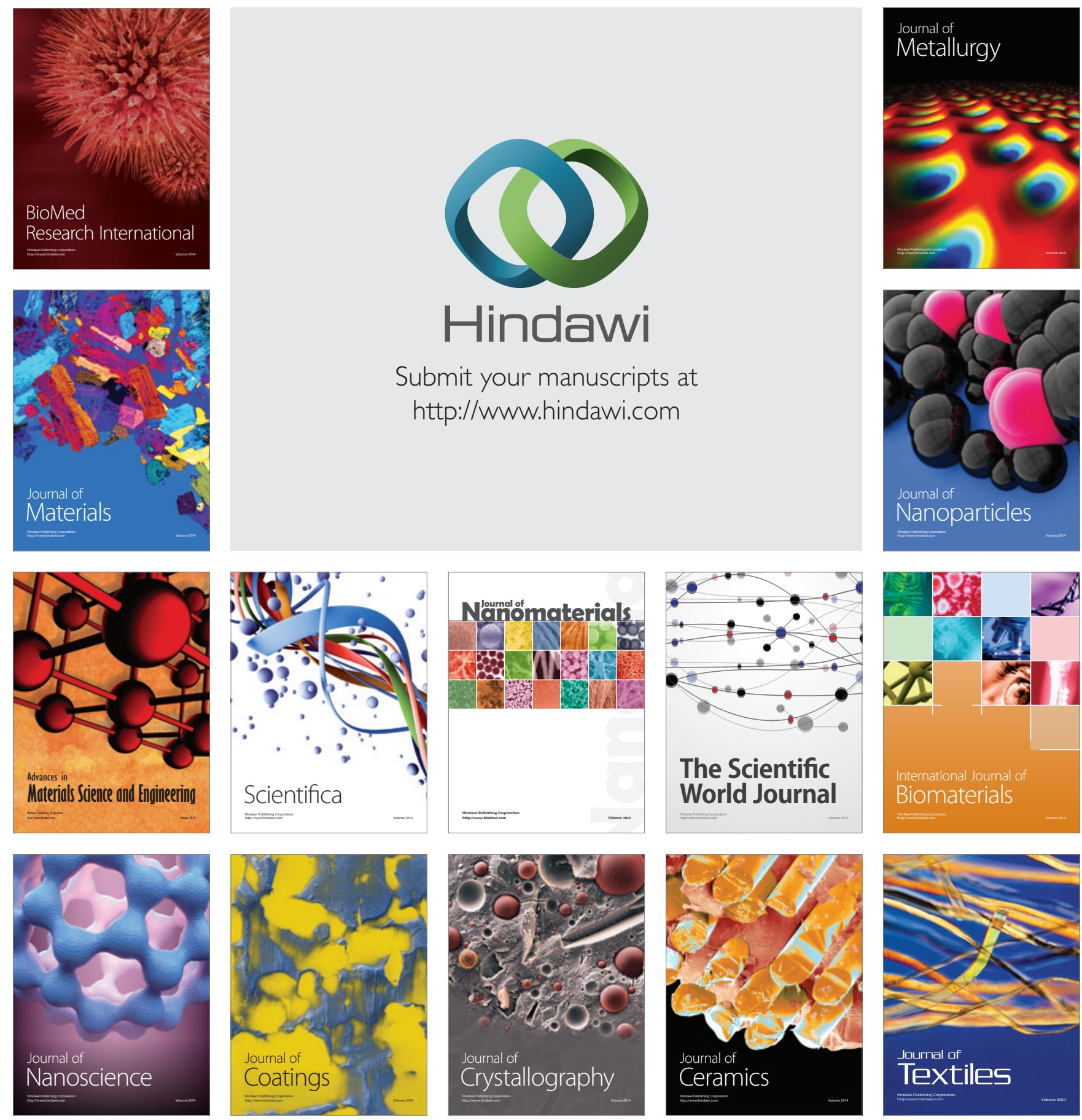